\title{
P01-153
}

\section{SEXUAL DYSFUNCTIONS IN ANXIOUS DEPRESSIVE DISORDERS}

\section{A. Tiugan}

Clinical Emergency Military Hospital, Craiova, Romania

Background: The ethiopathogenic mechanisms of depression and anxiety overlap at a certain moment on most part of those of the sexual dysfunction. Hypercorticolezemia from the anxious depressive disorders, with direct impact on the hypocampus (reducing the volume), through the two mechanisms (biological and organic) may generate and especially accentuate the dysfunctions in the sexual activity.

Objectives: In more than few cases, the anxious depressive disorders are accompanied by sexual dysfunctions, wh

During antidepressant therapy, especially with inhibitors specific for recapturing serotonine, different types of sexual dysfunctions may appear.

Methods: In a retrospective examination, out of 420 patients fulfilling the DSM IV R criteria for the anxious and depressive with anxious and depressive disorder, the presence of sexual dysfunction was highlighted in 340 patients $(80,9 \%)$. In 260 patients $(76,47 \%)$ the sexual dysfunction was present in the clinical board of anxiety and depression; in 78 patients (30\%) the sexual dysfunction accentuated during antidepressant therapy (predominantly with specific inhibitors for recapturing serotonin). In 80 patients (23,53\%), the anxious depressive symptomatology was preceded by sexual dysfunctions in various clinical forms. ich accentuate the anxiety and the depression.

Conclusions: The presence of sexual dysfunction in an anxious depressive board represents a negative predictive factor in the evolution of the affective disorder, delaying remission. The use of the mood stabilizer, by increasing GABA in the mezocortical circuit and equilibrating the GABA/DOPAMINE, can be beneficial for the improvement of the sexual dysfunction in the anxious depressive disorders. 